\title{
Design of Sliding Mode Controller with Auto-tuning Method
}

\author{
Wei He ${ }^{1}$, Yujia Zhai $^{2}$ \\ ${ }^{1}$ Automatic Control and System Engineering, University of Sheffield \\ Flat 19C, Opal 2, Brightmore House, 12 St. George's Close, Sheffield, S3 7HD, England \\ ${ }^{2}$ Department of Electrical and Electronics Engineering, Xi' an Jiaotong-Liverpool University, \\ Suzhou, China
}

(Tel : +86-512-1886-1415 E-mail: Sanghyuk.Lee@xjtlu.edu.cn)

Abstract Sliding mode control(SMC) are carried out in this literature. And to make the controllers perform better, fuzzy logic was chosen,it makes PID controller auto-tuning parameters and reduced the chattering problem of sliding mode control. Since SMC take error and derivative of error as inputs, after comparison some results are obtained.PID controller response faster yet sliding mode control is much steadier. However certain problems cannot be ignored that the chattering phenomenal cannot be reduced entirely and this motion may hurt the machine; this project only considered a simple system, there is no guarantee PID can work as well as in this case for a much more complex system. MATLAB simulink was the main approach to obtain the performance of the two controllers: to observe the control output of the two controllers, electric circuit and special controllers are designed and tested in MATLAB.

- Key words : Sliding Mode Controller, PID controller, Auto-tunning

\section{Introduction}

Generally, nonlinear system control has been carried out by way of feedback linearization with manifold theory [1], and PID controller for low order systems [1]. However, it was not easy to derive feedback linearization technique because it was needed mathematical background. Furthermore, PID controller was also needed tuning process [2]. In order to get rather easy implementation of nonlinear controller, sliding mode controller was proposed [3]. Yet a new ideal of control has been came up with at 1970s called sliding mode control and after the publication of a survey paper in the IEEE transactions on automatic control wrote by Vadim I. Utkin in 1977 [8], it draw a lot of interesting from researchers worldwide. The most significant feature is that the structure of the control could change during the transientprocess: different control law will be applied according to the current state of the system; the rules that to switch control laws are determined by the current value of the error and its derivative. Control laws are usually continuous control functions yet the switch action makes the control discontinuously. And since the control only depends on the state of the system, sliding mode control is totally insensitive to external disturbance which leads to a very high robust quality. However the discontinuously characteristic also caused a serious chattering problem which could damage machines; thus the core attention on sliding mode so far is to reduce the chattering phenomenal. In this 
project fuzzy logic is considered to achieve this aim.

In this paper, we considered SMC with fuzzy logic, in which conventional binary SMC inputs are replaced with linear component with the help of fuzzy logic $\cdots$ Control performance was compared with auto-tuning PID controller. Normally, PID controller is so far the most widely used controller in practice. According to a survey conducted by the Japan Electrical Measuring Instrument Manufacture's Association in 1989, it said more than $90 \%$ of the control loops in control system were PID type controllers [5]. It has simple structure and therefore can be applied into varieties of situations with high robust. The controller has several important functions: provide feedback of the control system; reduce steady-state error and predict trend of change and take control action in advance. These functions are achieved by its structure: three different terms work individually and give an output signal together. After the long time updating, the controller have survived many changes in technology for a wild range of filed in industry. In this project, an auto-tuning PID controller is studied. For auto-tuning case, the parameters of the controller could adjust automatically based on the current state of the system, and this update is a continuous action. To realize the auto-tuning function, fuzzy logic will be used during the control.

This paper is organized as follows. In the next chapter, SMC was introduced. In chapter 3, fuzzy logic is introduced in both the two parts for their own adjustment. Following a comparison of the two controllers under asame objective system and discussion on the result and suggested future works are provided in the last part.

\section{Sliding mode control (SMC)}

Sliding mode control is a nonlinear control method that can force the system to "slide" along a specific surface of the system's normal behavior. It is generally regarded as very robust and simple to implement; however the so-called "chattering phenomenal (that the control would oscillation)"could make serious damage to machine [6]. Still the theory becomes one of the most significant research topics in the control engineering domain.

In sliding mode control, the structure of the system would switch from one continuous structure to another based on the current position in the state space as shown in equation (4), thus it is also known as variable structure control (VSC). The control laws are continuous functions yet the switch action makes the control discontinuous. The design problem then comes to be selection of parameters for the two structures and define the switching manifold [7].

$$
u= \begin{cases}u^{+}(x, t) & \text { if } s(x)>0 \\ u^{-}(x, t) & \text { if } s(x)>0\end{cases}
$$

\subsection{Stability}

Consider a system

$$
\begin{aligned}
& \dot{x}_{i}=x_{i+1} \\
& \dot{x}_{n}=-\sum_{i=1}^{n} a_{i} x_{i}+f(x)+u
\end{aligned}
$$

Where $\mathrm{u}$ is system input, $\mathrm{f}(\mathrm{x})$ is disturbance $a_{i}$ are constant, and system output $y=x_{1}$.

Hyper plane is expressed by

$$
s=S(x)
$$

Then, $\mathrm{c}_{\mathrm{i}}$ is constant and $\mathrm{C}_{\mathrm{n}}=1$. Set the plane $\mathrm{s}=0$

$$
\dot{x}_{i}=-\sum_{i=1}^{n-1} c_{i} x_{i}
$$

Which could leads to the resulting equation of the sliding mode control:

$$
\dot{x}_{n-1}=-\sum_{i=1}^{n-1} c_{i} x_{i}
$$


This equation shows the sliding mode only depends on parameters $c_{i}$ and outside disturbance has nothing to do with the control structure. This means that sliding mode control is of very high robust.

\subsection{Sufficient condition}

When a moving point reaches the area around sliding manifold, in order to make the point move alone the surface, it must obey the following pair of inequalities:

$$
\lim _{s \rightarrow 0^{+}} \dot{s} \leq 0 \text { and } \lim _{s \rightarrow 0^{-}} \dot{s} \geq 0
$$

Which can be written as

$$
\lim _{s \rightarrow 0} s \dot{s}<0
$$

Assume any point that doesn't near the sliding surface. It must be driven to move towards the sliding manifold to realize the control action, thus the sufficient condition can be obtained as

$$
s \dot{s}<0
$$

where

$$
\dot{s}=\frac{d s}{d x} * \frac{d x}{d t}=\frac{d s}{d x} * \dot{x}=\frac{d s}{d x} * f(x, t)+B(x, t) u
$$

\subsection{Design of sliding manifold}

Assume a general linear system

$$
\dot{x}=A x+b u
$$

The sliding manifold is usually in the form of

$$
\boldsymbol{s}(\boldsymbol{x})=\boldsymbol{C}^{T} \boldsymbol{x}=\sum_{i=1}^{n} c_{i} x_{i}=\sum_{i=1}^{n-1} c_{i} x_{i}+x_{n}
$$

Where $\boldsymbol{C}=\left[\begin{array}{lll}c_{1} \ldots & c_{n-1} & 1\end{array}\right]^{T}$ and should be Hurwitz; $\boldsymbol{x}$ is state space, and $\dot{x}_{i}$ obeys equation $(5,6)$.

Chattering is oscillation motion of the control state near sliding surface. According to K. David Young [3], there are two possible reasons that produce chattering motion. First is the non-ideality of switching, for example delay. The parasitic dynamics in series with the plant can cause an oscillation that of small amplitude but high frequency around the sliding surface. The interaction between parasitic dynamics and Variable Structure Control produce a non-decaying oscillatory component that owns finite amplitude and frequency. This is usually called chattering.

Secondly, the non-idealities characteristic of the switching alone can produce a high frequency oscillation and this mainly because of time delay, which makes it realistic that discrete-time control design techniques can be applied to decrease switch delays.

In practice control cases, both two causes mentioned above exist. Young introduced a so called piecewise linear or smooth approximation of the switching component in a boundary layer of the sliding surface that is said to be the most commonly used approach that can reduce the effect of chattering problem.

Vadim I. Utkin introduced a formal technique to find equations for ideal sliding mode control that is called "equivalent control method" [8]. By setting derivative of vector $\mathrm{s}(\mathrm{x})$ to zero, the resulting algebraic system can be solved for control vector. Assume a linear system with respect to control,

$$
\dot{x}=f(x, t)+B(x, t) u
$$

Where $u$ can be determined by (8). Set (12) to be 0 ,

$$
\dot{s}=\frac{d s}{d x} *(f(x, t)+B(x, t) u)=0
$$

and set $G=\frac{d s}{d x}$, the equivalent control can be derivative as 


$$
u_{e q}=-(G B)^{-1} G f
$$

then substitute (13) into (11) can get the ideal sliding mode function

$$
\left\{\begin{array}{c}
\dot{x}=f-B(G B)^{-1} G f \\
s=0
\end{array}\right.
$$

\section{Design and simulation}

The block of simulink of a traditional sliding mode control is usually of the following form:

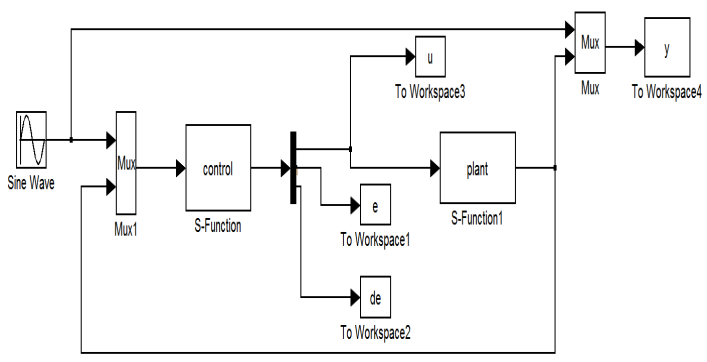

[Fig. 1] Structure of a traditional SMC

And a typical phase trajectory of the output is shown as

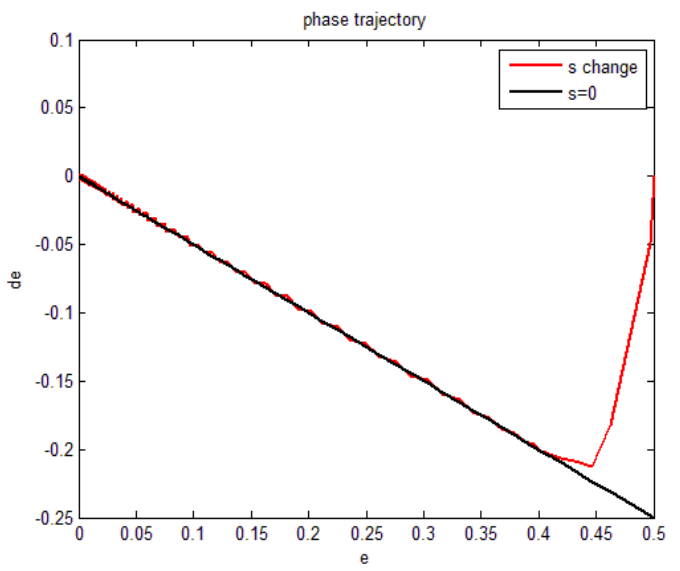

[Fig. 1] Typical phase trajectory of SMC Consider an objective system

$$
\ddot{\theta}=f(\theta, \theta)+b u(t)+E(t)
$$

Where $\ddot{\theta}_{\text {is }}$ the angular acceleration of the body, $f$ is known, $u$ is control input and $E(t)$ is unknown disturbance, and set the maximum value of disturbance is $\mathrm{D}$.

According to (19), sliding manifold is therefore

$$
s(t)=c e(t)+\dot{\theta}(t), c>0
$$

Set

$$
\begin{aligned}
& e(t)=\theta_{d}(t)-\theta(t), \\
& \dot{\theta}=\theta_{d}(t)-\theta(t)
\end{aligned}
$$

$\Theta_{\mathrm{d}}$ is set angle and $\Theta$ is output angle.

Control rule is designed as

$$
u=\frac{1}{b}(-f(\theta)+\ddot{\theta}+c \dot{\theta}+K(t) \operatorname{sgn}(s))
$$

Where $\operatorname{sgn}(s)$ represents the sign of $s$, i.e.

$$
\operatorname{sgn}(s)= \begin{cases}-1 & \text { if } s<0 \\ 0 & \text { if } s=0 \\ 1 & \text { if } s>0\end{cases}
$$

$$
\begin{aligned}
& \text { And } \begin{aligned}
& K(t)=\max |E(t)|+\eta, \eta>0 . \\
& s \dot{s}=s(\ddot{\theta}+c \dot{\theta})=s\left(\ddot{\theta}_{d}-\ddot{\theta}+c \dot{\theta}\right) \\
&=s\left(\ddot{\theta}_{d}-f(\theta, \theta)-b u(t)\right. \\
&-E(t)
\end{aligned}
\end{aligned}
$$

It can get

$$
\begin{aligned}
s \dot{s} & =s(-K(t) \operatorname{sgn}(s)-E(t)) \\
& =-K(t)|s|-E(t) s \leq-\eta|s|
\end{aligned}
$$

In (20) it can be seen that $\mathrm{K}(\mathrm{t})$ is the parameter for different control law part, so it is the reason that may 
cause chattering. In this case, $\mathrm{K}(\mathrm{t})$ is adjusted by fuzzy logic to reduce the oscillation.

As introduced before, the sufficient conditions of sliding mode is to make $s \dot{s} \leq 0$,

So to guarantee the stability of the control, fuzzy rules are designed as follows:

If $s \dot{s}>0, \mathrm{~K}(\mathrm{t})$ should increase;

if $s \dot{s}<0, \mathrm{~K}(\mathrm{t})$ should decrease.

That is to say the fuzzy rules can be expressed as: If $s \dot{s}>0, \Delta \mathrm{K}>0$;

If $s \dot{s}<0, \Delta \mathrm{K}<0$.

Define the fuzzy set of input $s \dot{s}$ and output $\Delta \mathrm{K}$ :

$$
\begin{aligned}
& s \dot{s}=\{\mathrm{NB} \text { NM ZO PM PB }\} \\
& \Delta \mathrm{K}=\{\mathrm{NB} \text { NM ZO PM PB },
\end{aligned}
$$

Where NB means negative big, NM is middle negative, $\mathrm{ZO}$ is zero, $\mathrm{PM}$ is perfect middle, and $\mathrm{PB}$ is positive big.

Thus the fuzzy rules are designed as follows:

IF ss' is $\mathrm{NB}$, THEN $\Delta \mathrm{K}$ is $\mathrm{NB}$;

IF $s s^{\prime}$ is NM, THEN $\Delta \mathrm{K}$ is NM;

IF $s s^{\prime}$ is ZO, THEN $\Delta \mathrm{K}$ is ZO;

IF $s \dot{s}$ is PM, THEN $\Delta \mathrm{K}$ is PM;

IF $s \dot{s}$ is $\mathrm{PB}$, THEN $\Delta \mathrm{K}$ is $\mathrm{PB}$.

And the resulting membership functions are

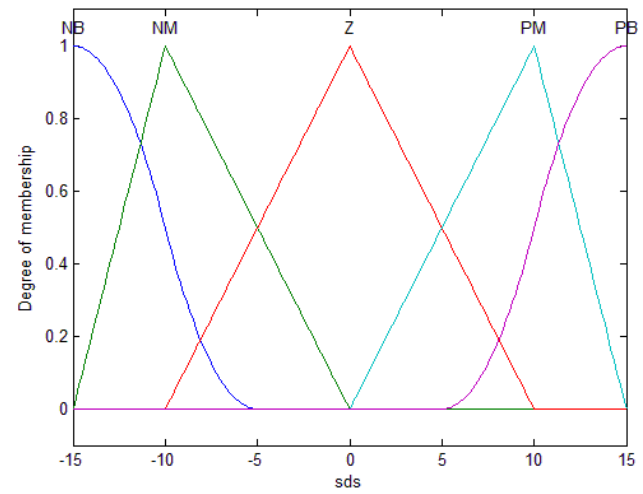

[Fig. 3] Membership function of input

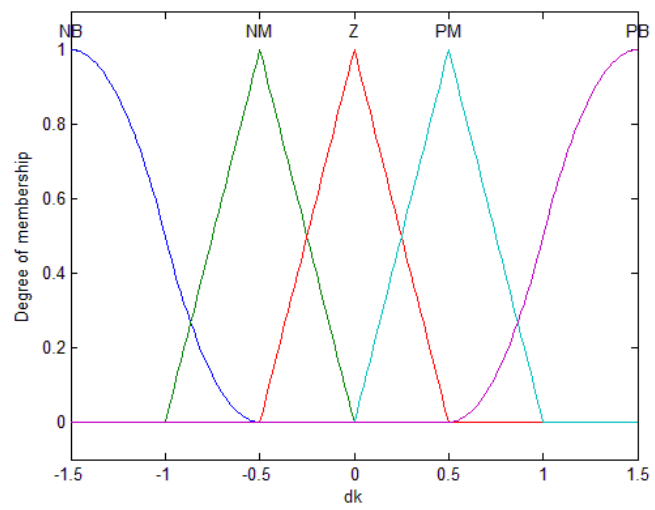

[Fig. 4] Membership function of output

The Simulink block is designed as follows:

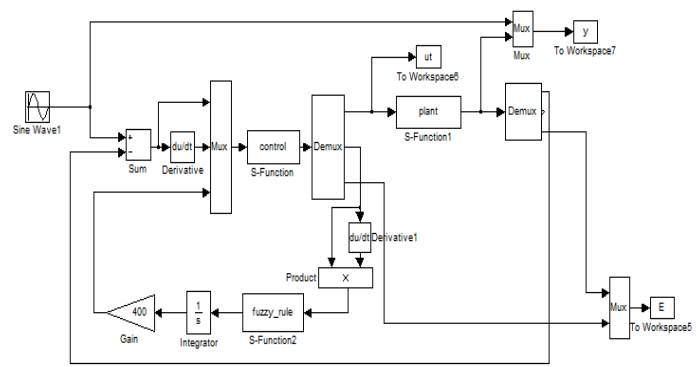

[Fig. 5] Structure of fuzzy sliding mode control

To investigate the control effect of fuzzy rules, the same system is tested without fuzzy logic, and results are shown below. The output error of traditional control (without fuzzy logic):

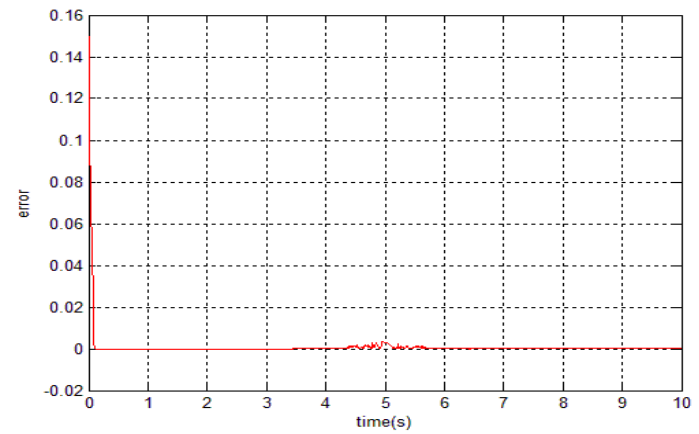

[Fig. 6] Error of traditional SMC 
Derivative of error above:

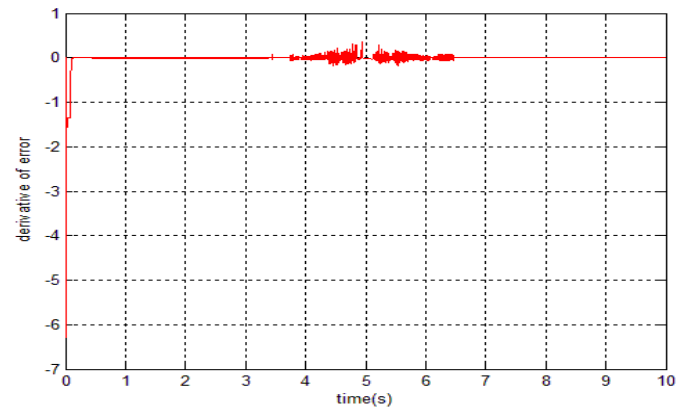

[Fig. 7] Derivative of error of traditional SMC

Output error by fuzzy involved SMC:

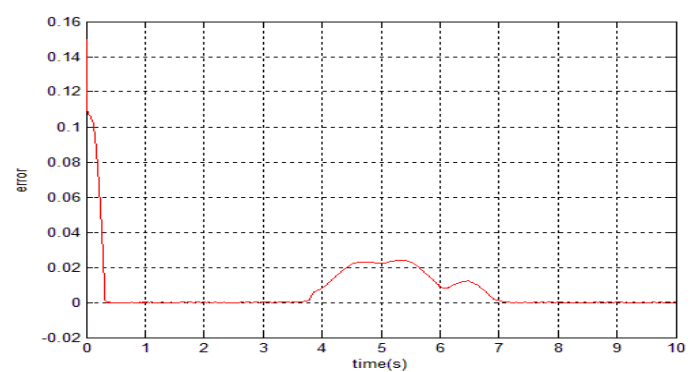

[Fig. 8] Error of fuzzy SMC

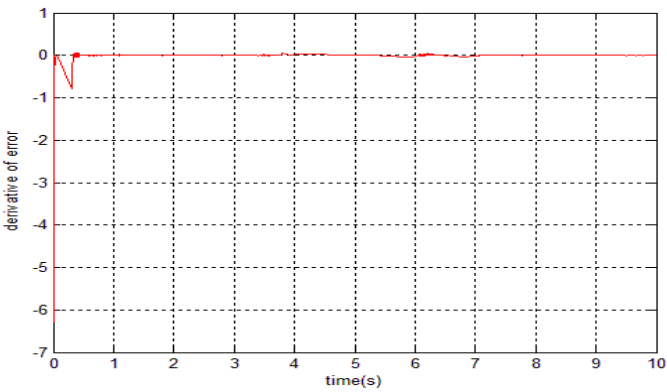

[Fig. 9] Derivative of error of fuzzy SMC

To test the reaction of unexpected interrupt, a Gaussian shape disturbance is added to the system which centered at 5 second.

After the comparison of the error outputs provided, it can be seen clearly that with fuzzy logic involves in, the system is steadier when disturbance occurs. Compared to Figure 7, a much smoother wave appears in Figure 8at the same time near 5 second. The fuzzy logic does reduce the chattering problem greatly. The derivative in Figure 9shows an intense oscillation which indicates error changed fast and greatly. However the latter case also slows the response of the system: error reaches 0 much faster in Figure 6 than 8 and the amplitude of wave in Figure 7 is more than 0.02 while it is quite small in Figure 9 .

\section{Comparison with SMC and auto- tuning PID}

Both controllers take error e and derivative de as inputs. Put the two controllers into the same second order system that with the transfer function as below:

$G=\frac{100}{s^{2}+25 s}$

and the results are obtained as follows:

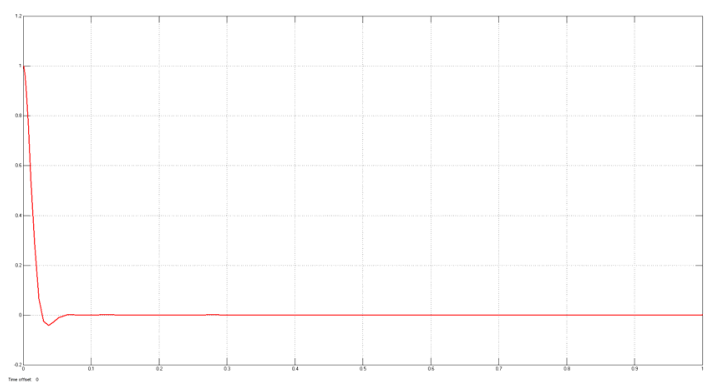

[Fig. 10] Error of PID

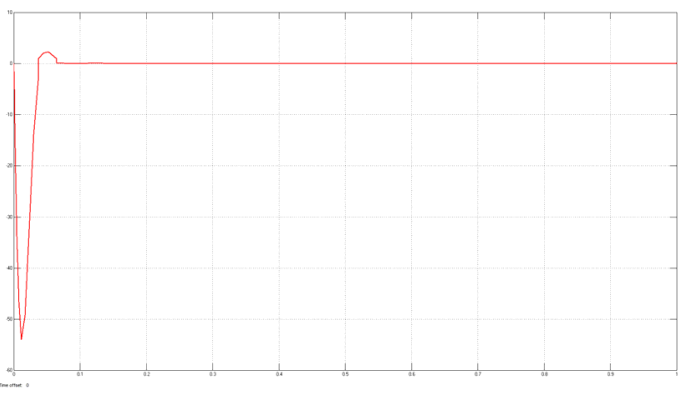

[Fig. 11] Derivative of error of PID 


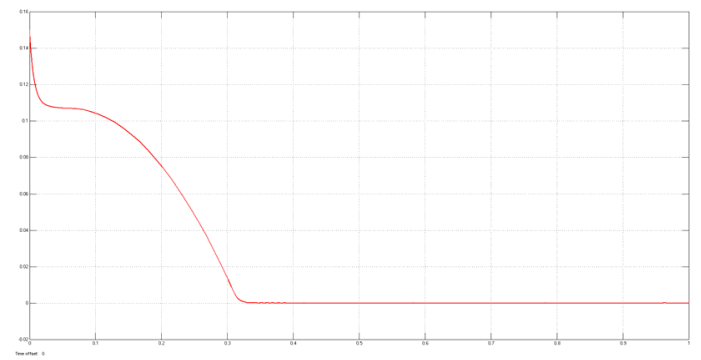

[Fig. 12] Error of SMC

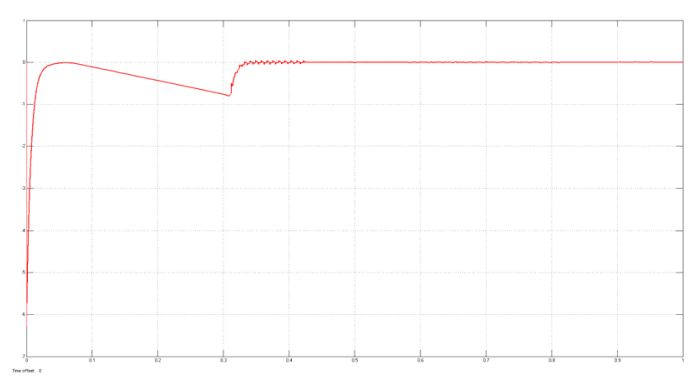

[Fig. 13] Dderivative of error of SMC

Comparing the figures in last section, several conclusions can be obtained as follows:

Firstly, PID controller responses faster than sliding mode control. Figure 10shows that the system get to zero steady state error before 0.1 second; while in Figure 12, sliding mode control uses more than 0.3 second to get a perfect control output.

However it is noticeable that the change rate of sliding mode is steadier than the other one. There is a sign change in Figure 11 for derivative of error that means output of PID control experiences an overshoot while the other one has no overshot at all. Plus, the range of error also differs from each other: for PID case, error decreases from 1 to negative then slowly approaches zero; yet in sliding mode control, error ranges from 0.16 to 0 .This also represents a steady control effect.

Although fuzzy is involved in sliding mode control, chattering problem still exists, especially during 0.3 to 0.4 second; Figure 13 clearly shows an oscillation in the change of error. And after zero steady state error, oscillations appear occasionally.

\section{Conclusion}

The target of the project is to compare two different approaches controllers: PID controllers and sliding mode control. Both the two controllers take error and derivative of error as inputs make it meaningful to compare outputs of the two controllers to see which is better.To do so, the two controllers were studied individually in details.

Sliding mode hasn't raised a lot of interesting until a Russian engineering Vtkin published his ideal of the control in 1977. In this control, structures can switch from one continuous function to another which makes the control action discontinuous. Due to this feature sliding mode control is very insensitive to external disturbance and isof high robust.Nevertheless, this switch motion also causes a serious chattering problem that the system could oscillation during the control procedure and may damage engine for this reason. This project applied fuzzy logic into the control system to reduced the chattering phenomenaland the result showed great improvement compared to a traditional sliding mode control output. At the same time however, fuzzy sliding mode slowed the response of the control system according to the output.

Then in section 4, a simple second order system was applied into both the two controllers. Outputs were displayed, and after comparison, some conclusions were obtained:

1. PID controller response faster than sliding mode control

2. Fuzzy sliding mode control is steadier than PID control.

This conclusion can be used into practice: when a control requires fast control and do not require accuracy, PID is preferred like air-conditioner; when a control need steady control and is not urgent about time, sliding mode control is a better choice.

This is just a roughly comparison between fuzzy 
auto-tuning PID controller and fuzzy sliding mode control on a simple second order system. To get an overall and exact comparison of the two controllers many other works remain to be complete.

\section{REFERENCES}

[1] C. P. Coleman and D. Godbole, "A Comparison of Robusteness: Fuzzy Logic, PID, and Sliding Mode Control," in IEEE World Congress on Computational Intelligence., Walt Disney, Vol. 3, pp. 1654-1659, 1994.

[2] Fuzzy logic [online]. Available: http://en.wikipedia. org/wiki/Fuzzy_logic.

[3] K. D. Young, "A Control Engineer's Guide to Sliding Mode Control," IEEE Trans. Control Systems Technology, Vol. 7, No. 3, pp. 328-342, 2002.

[4] K. Johan and T. Hägglund, Advanced PID Control. New York: ISA-The Instrumentation, Systems, and Automation Society, 2006.

[5] L. Yamamoto and L. Hashimoto, "Present status and Future Needs: the view from Japanese industry," in Chemical Process Control-CPCIV: Proc.4th Int. Conf. on Chemical Process Control, eds, Texas, pp, 1-28, 1991.

[6] V. I. Utkin, "Application Oriented Trends in Sliding Mode Control Theory", in Industrial Electronics, Control, and Instrumentation, Maui, HI, Vol. 3, pp. 1937-1942, 1993.

[7] V. I. Utkin, "Sliding Mode Control", UNESCOEOLSS, Control Systems, Robotics and Automation., vol. XII.

[8] V. I. Utkin, "Variable Structure System With Sliding Modes," IEEE Trans. Automat. Contr., Vol. AC-22, pp. 212-222, 1997.

[9] W. D. Chang, R. C. Hwang, and J. G. Hsieh, "An auto-tuning PID control for a class of nonlinear systems based on Lyapunov approach," J. Process Control, Vol. 12, pp. 233-242, 2002.
[10] Y. Huang and S.Yasunobu, "A General Practical Design Method For Fuzzy PID Control From Conventional PID Control," in Proc. IEEE Int. Conf. Fuzzy Systems, San Antonio, TX, Vol. 2, pp. 969-972, 2000.

[11] Z. M. Chen, Sliding mode variable structure control theory and application. Beijing: Publishing House of Electronics Industry, 2012 (in Chinese).

\section{저자소개}

He Wei

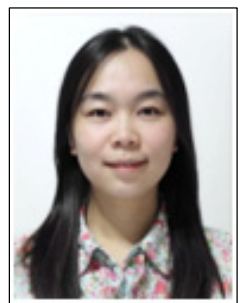

- 2009. Sep. : Dept. of Electrical Electronic Engineering, Xi'an-Jiaotong Liverpool University (BEng) - 2013. Sep. Present : Dept. of Automatic Control and Systems Engineering, University of Sheffield (Msc(Eng))

•E-Mail : he.wei.ellen@gmail.com

Yujia Zhai

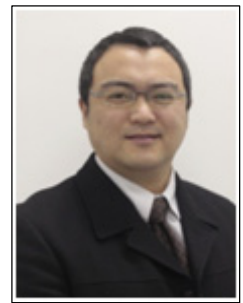

- 2001. Department of Electrical Engineering, Changchun University (Bachelor of Eng.)

- 2004. Department of Electrical Engineering and Electronics, University of Liverpool (UoL) (Master in Information and Intelligence Engineering) - 2009. Liverpool John Moores University (LJMU) (Ph.D. in Control Engineering)

-E-Mail : yujia.zhai@xjtlu.edu.cn

$<$ Research Interest> : Nonlinear Control and Robustness, Automotive Engine Modeling and Dynamics, Analysis Artificial Intelligence 